GA-A24208

\title{
MODEL OF THE TOKAMAK EDGE DENSITY PEDESTAL INCLUDING DIFFUSIVE NEUTRALS
}

\author{
by \\ K. H. Burrell
}




\section{DISCLAIMER}

This report was prepared as an account of work sponsored by an agency of the United States Government. Neither the United States Government nor any agency thereof, nor any of their employees, makes any warranty, express or implied, or assumes any legal liability or responsibility for the accuracy, completeness, or usefulness of any information, apparatus, product, or process disclosed, or represents that its use would not infringe privately owned rights. Reference herein to any specific commercial product, process, or service by trade name, trademark, manufacturer, or otherwise, does not necessarily constitute or imply its endorsement, recommendation, or favoring by the United States Government or any agency thereof. The views and opinions of authors expressed herein do not necessarily state or reflect those of the United States Government or any agency thereof. 
GA-A24208

\title{
MODEL OF THE TOKAMAK EDGE DENSITY PEDESTAL INCLUDING DIFFUSIVE NEUTRALS
}

\author{
by \\ K. H. Burrell
}

This is a preprint of a paper to be published in Physics of Plasmas.

General Atomics, P.O. Box 85608, San Diego, California 92186-5608 USA

\author{
Work supported by \\ U.S. Department of Energy under \\ Contract DE-AC03-99ER54463
}

GENERAL ATOMICS PROJECT 30033

JANUARY 2003 


\section{ABSTRACT}

Several previous analytic models of the tokamak edge density pedestal have been based on diffusive transport of plasma plus free-streaming of neutrals. This latter neutral model includes only the effect of ionization and neglects charge exchange. The present work models the edge density pedestal using diffusive transport for both the plasma and the neutrals. In contrast to the free-streaming model, a diffusion model for the neutrals includes the effect of both charge exchange and ionization and is valid when charge exchange is the dominant interaction. Surprisingly, the functional forms for the electron and neutral density profiles from the present calculation are identical to the results of the previous analytic models. There are some differences in the detailed definition of various parameters in the solution. For experimentally relevant cases where ionization and charge exchange rate are comparable, both models predict approximately the same width for the edge density pedestal. 
Experimental observations [1-3] and theoretical modeling [4] both show that the $\mathrm{H}$ (high)-mode pedestal parameters in tokamak discharges have a substantial impact on the performance of the core plasma. Accordingly, developing a predictive understanding of the pedestal parameters is quite important. Recent work on the edge density pedestal indicates that the transport of neutral hydrogen plays a significant role in determining the shape of the edge density profile [5-7]. Utilizing a simple, free-streaming model of the neutral penetration [8-10], the width of the steep gradient region at the plasma edge is predicted to be the ionization mean free path for neutrals. The results of this simple model compare quite favorably with experimental measurements of this width and with experimental measurements of the maximum density gradient at the plasma edge $[6,7]$. The agreement strongly suggests that atomic physics is important in the physics of the edge density pedestal. The results of the much more elaborate UEDGE code also agree reasonably well with the experimental results [11], again supporting the importance of the atomic physics of hydrogen neutrals in setting the width of the edge density pedestal.

Although the agreement of the UEDGE results with the simpler analytic model is heartening, this agreement actually poses a significant physics puzzle. The neutral model included in UEDGE is a diffusion model [12]; diffusion models for neutral transport are valid when charge exchange events are much more frequent than ionization events [13-15]. However, the freestreaming model presented in Refs. [8-10] includes only the effect of ionization and completely neglects charge exchange. As is shown in Fig. 1, for the experimentally relevant case of a deuterium plasma, the charge exchange reaction rate is always comparable to or greater than the electron impaction ionization rate for the usual experimental conditions where the ion temperature is greater than or equal to the electron temperature. Since charge exchange is the dominant atomic physic interaction for deuterons, how is it that a model which neglects charge exchange compares so well with experiment? 
The present work demonstrates why the calculations using free-streaming and diffusion models for the neutrals give approximately the same result. As will be shown, an analytic solution exists to the coupled neutral-electron diffusion problem. This solution has two important features. First, it has the same functional form as the solution obtained using the free-streaming neutral model [5-10]. Second, although the parameter which governs the width of the steep gradient region at the plasma edge is different than that for the free-streaming model, it has approximately the same numerical value for deuterium plasmas in the temperature range used in the experiments. Accordingly, this explains why the two models give approximately the same result, even though they are based on quite different physics.

Using a one dimension model as the previous work [5-10], I consider the coupled continuity equations for electrons and neutrals.

$$
\begin{aligned}
& \frac{d \Gamma_{e}}{d x}=n_{e} n_{o}\left\langle\sigma_{i} \mathrm{v}_{e}\right\rangle \\
& \frac{d \Gamma_{o}}{d x}=-n_{e} n_{o}\left\langle\sigma_{i} \mathrm{v}_{e}\right\rangle .
\end{aligned}
$$

Here, $\Gamma$ is the particle flux, $n$ is the particle number density, the subscripts $e$ and $o$ denote electrons and neutrals, respectively, and $\left\langle\sigma_{i} v_{e}\right\rangle$ is the neutral ionization rate due to electron impact averaged over the electron velocity distribution.

As in the previous work [5-10], I assume a diffusive model for the electron particle transport with $\Gamma_{e}=-D_{e} d n_{e} / d x . D_{e}$ is taken to be constant. Unlike the previous work, I assume a diffusive model for the neutrals, $\Gamma_{o}=-D_{o} d n_{o} / d x$. I use the UEDGE form for $D_{o}=\mathrm{v}_{t h}^{2} /\left[2 n_{e}\left(\left\langle\sigma_{c x} \mathrm{v}_{i}\right\rangle+\left\langle\sigma_{i} \mathrm{v}_{e}\right\rangle\right)\right]$ [see Eq. (A8) in Ref. 12]. Here, $\mathrm{v}_{t h}=\left(2 T_{i} / m_{i}\right)^{1 / 2}$ is the ion thermal speed, $T_{i}$ is the ion temperature, $m_{i}$ is the ion mass and $\left\langle\sigma_{c x} v_{i}\right\rangle$ is the charge exchange rate averaged over the ion velocity distribution. The form for $D_{o}$ has been specialized to the present case of a pure hydrogenic plasma with equal electron and ion number densities. A diffusion approximation makes sense only when the gradient scale lengths are long compared to neutral mean free paths. 
I will solve this problem on the half space $0 \leq x<\infty$. The boundary conditions as $x \rightarrow \infty$ are $n_{e} \rightarrow n_{e}(\infty), n_{o} \rightarrow 0, \Gamma_{e} \rightarrow 0$ and $\Gamma_{o} \rightarrow 0$

Summing Eqs. (1) and (2), integrating and applying the boundary condition as $x \rightarrow \infty$ yields $\Gamma_{e}+\Gamma_{o}=0$. Using the diffusion equations, this result can be rewritten as

$$
D_{e} \frac{d n_{e}}{d x}+\frac{\mathrm{v}_{t h}^{2}}{2 n_{e}\left(\left\langle\sigma_{c x} \mathrm{v}_{i}\right\rangle+\left\langle\sigma_{i} \mathrm{v}_{e}\right\rangle\right)} \frac{d n_{o}}{d x}=0 .
$$

In order to proceed further analytically, I assume, as was done previously [5-10], that the electron and ion temperatures are constant, thus making the reaction rates $\left\langle\sigma_{c x} \mathrm{v}_{i}\right\rangle$ and $\left\langle\sigma_{i} \mathrm{v}_{e}\right\rangle$ constant. If Eq. (3) is multiplied by $n_{e}$, it can be written as an exact derivative. Integrating this and using the boundary condition as $x \rightarrow \infty$ yields

$$
n_{o}(x)=\frac{D_{e}\left(\left\langle\sigma_{c x} \mathrm{v}_{i}\right\rangle+\left\langle\sigma_{i} \mathrm{v}_{e}\right\rangle\right)}{\mathrm{v}_{t h}{ }^{2}}\left[n_{e}{ }^{2}(\infty)-n_{e}{ }^{2}(x)\right] \text {. }
$$

Now substitute Eq. (4) and $\Gamma_{e}=-D_{e} d n_{e} / d x$ into Eq. (1). Note that both sides of the resulting equation are proportional to $D_{e}$; hence, it can be divided out, yielding

$$
\frac{d^{2} n_{e}}{d x^{2}}=-\frac{\left\langle\sigma_{i} v_{e}\right\rangle\left(\left\langle\sigma_{c x} \mathrm{v}_{i}\right\rangle+\left\langle\sigma_{i} \mathrm{v}_{e}\right\rangle\right)}{\mathrm{v}_{t h}{ }^{2}} n_{e}\left[n_{e}{ }^{2}(\infty)-n_{e}{ }^{2}\right] .
$$

If Eq. (5) is multiplied by $d n_{e} / d x$, both sides become exact differentials, which can be integrated to give

$$
\frac{d n_{e}}{d x}=\frac{\left\langle\sigma_{i} \mathrm{v}_{e}\right\rangle^{1 / 2}\left(\left\langle\sigma_{c x} \mathrm{v}_{i}\right\rangle+\left\langle\sigma_{i} \mathrm{v}_{e}\right\rangle\right)^{1 / 2}}{\sqrt{2} \mathrm{v}_{t h}}\left[n_{e}{ }^{2}(\infty)-n_{e}{ }^{2}\right] .
$$

The solution to this first order equation is

$$
n_{e}(x)=n_{e}(\infty) \tanh \left(\frac{x}{\lambda}+c\right)
$$

where

$$
\lambda=\frac{\mathrm{v}_{t h}}{n_{e}(\infty)}\left[\frac{2}{\left\langle\sigma_{i} \mathrm{v}_{e}\right\rangle\left(\left\langle\sigma_{c x} \mathrm{v}_{i}\right\rangle+\left\langle\sigma_{i} \mathrm{v}_{e}\right\rangle\right)}\right]^{1 / 2} .
$$


The integration constant $c$ can be determined from an as yet unspecified boundary condition at, for example, $x=0$.

The solution is completed by using Eq. (7) in Eq. (4) to produce

$$
n_{o}(x)=\frac{D_{e}\left(\left\langle\sigma_{c x} \mathrm{v}_{i}\right\rangle+\left\langle\sigma_{i} \mathrm{v}_{e}\right\rangle\right)}{\mathrm{v}_{t h}^{2}} \frac{n_{e}^{2}(\infty)}{\cosh ^{2}\left(\frac{x}{\lambda}+c\right)}
$$

The free streaming neutral model produces a solution with the same functional form as Eqs. (7) and (9). The differences with the present model lie in the expression for $\lambda$ and the form of the first factor on the right hand side of Eq. (9). For the free-streaming solution, the expression for the scale length is $\lambda_{F S}=\mathrm{v}_{t h} /\left[n_{e}(\infty)\left\langle\sigma_{i} \mathrm{v}_{e}\right\rangle\right]$ Accordingly, the ratio of the two scale lengths is

$$
\frac{\lambda}{\lambda_{F S}}=\left[\frac{2\left\langle\sigma_{i} \mathrm{v}_{e}\right\rangle}{\left\langle\sigma_{c x} \mathrm{v}_{i}\right\rangle+\left\langle\sigma_{i} \mathrm{v}_{e}\right\rangle}\right]^{1 / 2} \text {. }
$$

As shown in Fig. 1 for a deuterium plasma, the ionization and charge exchange rate coefficients are roughly equal in the 40 to $200 \mathrm{eV}$ temperature range in which the previous work is stated to be valid $[6,7]$. Under these conditions, the ratio $\lambda_{/} \lambda_{F S}$ is close to unity. Accordingly, it is now clear why the previous, simple analytic model and the UEDGE results were in approximate agreement even though significantly different physics was used in the two models.

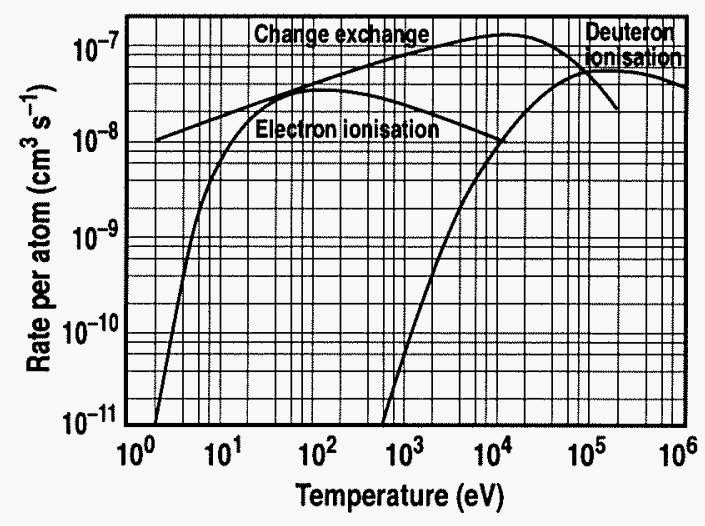

Fig. 1. Maxwellian averaged rate coefficients for neutral deuterium atoms. Shown are the electron impaction ionization rate coefficient, the deuteron-deuterium charge exchange rate and the deuteron-deuterium ion impact ionization rate coefficient as a function of electron temperature (for electron ionization) or ion temperature (for deuteron-deuterium interactions). Rate coefficients are from Freeman and Jones [16]. 
Figure 1 suggests that the free-streaming model, with its complete neglect of charge exchange, does not contain all of the essential physics. Since the charge exchange rate is comparable or greater than the ionization rate, the charge exchange effects should be included in the model of the edge density pedestal. Indeed, as the edge temperatures approach $1 \mathrm{keV}$, Fig. 1 indicates that the free-streaming model would significantly overestimate the pedestal width.

Figure 1 also indicates that the diffusion approximation for the neutrals is only marginally valid for experimental conditions where temperature are in the 40 to $200 \mathrm{eV}$ range, since kinetic theory [13-15] demonstrates that the basic condition needed for validity of the diffusion approximation is $\left\langle\sigma_{c x} \mathrm{v}_{i}\right\rangle \gg\left\langle\sigma_{i} \mathrm{v}_{e}\right\rangle$ [13-15]. This inequality is more easily satisfied at higher edge temperatures or in the case, frequently seen experimentally, where the edge ion temperature significantly exceeds the edge electron temperature. The fact that the functional form for the solutions are identical for the cases $\left\langle\sigma_{c x} v_{i}\right\rangle \gg\left\langle\sigma_{i} v_{e}\right\rangle$ and $\left\langle\sigma_{c x} v_{i}\right\rangle<\left\langle\sigma_{i} v_{e}\right\rangle$ suggests that the functional form for the full kinetic solution will not be very different. However, to determine the proper expression for $\lambda$, a full kinetic treatment is really needed to properly include all the neutral physics in the edge pedestal model. Until that can be carried out, the present results are superior to those using the free-streaming model since the present results include the effects of both ionization and charge exchange. 


\section{REFERENCES}

[1] W. Suttrop, M. Kaufmann, H.J. de Blank, et al., Plasma Phys. Control. Fusion 39, 2051 (1997).

[2] T.H. Osborne, J.R. Ferron, R.J. Groebner, L.L. Lao, A.W. Leonard, R. Maingi, R.L. Miller, A.D. Turnbull, M.R. Wade, Plasma Phys. Control. Fusion 40, 845 (1998).

[3] M. Greenwald, R.L. Boivin, F. Bombarda, et al., Nucl. Fusion 37, 793 (1997).

[4] J.E. Kinsey, T. Onjun, G. Bateman, A. Kritz, A. Pankin, G.M. Staebler and R.E. Waltz, "Burning plasma projections using drift wave transport models and scalings for the H-mode pedestal," submitted to Nuclear Fusion.

[5] R.L. Boivin, J.A. Goetz, A.E. Hubbard, et al., Phys. Plasmas 7, 1919 (2000).

[6] R.J. Groebner, M.A. Mahdavi, A.W. Leonard, T.H. Osborne, G.D. Porter, R.J. Colchin and L.W. Owen, Phys. Plasmas 9, 2134 (2002).

[7] R.J. Groebner, et al. Plasma Phys. Control. Fusion 44, A265 (2002).

[8] F. Wagner and K. Lackner, Physics of Plasma-Wall Interactions in Controlled Fusion (D.E. Post and R. Behrisch, ed., Plenum Press, NY 1986) Series B, Physics Vol. 131, p. 931.

[9] W. Engelhardt and W.J. Feneberg, J. Nucl. Mater. 76-77, 518 (1978).

[10] M.A. Mahdavi, T.H. Osborne, A.W. Leonard et al., Nucl. Fusion 42, 52 (2002).

[11] N.S. Wolf, T.W. Petrie, G.D. Porter, T.D. Rognlien, R.J. Groebner, M.A. Mahdavi, "Role of neutrals in core fueling and pedestal structure in H-mode D III-D discharges," J. Nucl. Mater. (to be published).

[12] T.D. Rognlien, D.D. Ryutov, N. Mattor and G.D. Porter, Phys. Plasmas 6, 1851 (1999).

[13] J.W. Connor, Phys. Plasmas 19, 853 (1977).

[14] K.H. Burrell, Phys. Fluids 21, 2202 (1978).

[15] R.D. Hazeltine, M.D. Calvin, P.M. Valanju, E.R. Solano, Nucl. Fusion 32, 3 (1992). 
[16] R.J. Freeman and E.M. Jones, "Atomic Collision Processes in Plasma Physics Experiments: Analytic Expressions for Selected Cross Sections and Maxwellian Rate Coefficients, Culham Laboratory Report CLM-R 137 (H.M. Stationery Office, 1974). Copies can be ordered from The Stationery Office, P.O. Box 29, Norwitch, NR3 IGN, United Kingdom. 


\section{ACKNOWLEDGMENT}

Work supported by U.S. Department of Energy under Contract DE-AC03-99ER54463. 